ground between intrinsic ageing and the underlying causes of age-related multi-morbidity offers intriguing opportunities to improve the trajectories of health across our lengthening lifespans.

\section{APPLYING PERSONALISED MEDICINE IN CLINICAL PRACTICE}

Munir Pirmohamed. Wolfson Centre for Personalised Medicine, University of Liverpool, UK

10.1136/postgradmedj-2018-fpm.9

Personalised or precision medicine represents the overall concept of how drugs can be better targeted to individuals or groups of individuals to improve efficacy of the treatment, and minimize any safety issues, thereby improving the benefitrisk profile of the therapy. In order to achieve this goal, many different technologies will need to be utilised which includes all the omics technologies, drug pharmacokinetics and pharmacodynamics, and the use of wearable sensors, to name a few. While there has been a lot of emphasis on all omics technologies, genomics has had the greatest impact. Personalised or precision medicine approaches can be applied to chemical entities, cell therapies and nucleic acid-based therapies. For example, with respect to the latter, advances in gene therapy are beginning to produce real benefits in inherited diseases such as haemophilia, while antisense therapies are being utilised in a number of CNS conditions including spinal muscular atrophy and Huntington's disease. Targeted therapies developed on the basis of the identification of somatic driver mutations are now the norm rather than the exception in cancer medicine, with combinations of treatments including targeted and immune therapies promising tractable responses in previously untreatable malignancies. Pharmacogenomics is also gaining traction with different approaches being used including (a) developing or repurposing drugs on the basis of mutations in disease-causing genes; for example the use of ivacaftor in cystic fibrosis; (b) defining inter-individual variability in dose, for example with warfarin; and (c) in preventing serious adverse drug reactions; for example the use of HLA genotyping to prevent life-threatening adverse reactions such as toxic epidermal necrolysis. Pre-emptive genotyping approaches are also being used where genetic data is available at the point of prescribing, much like the availability of liver and renal function tests. It is important that future generations of healthcare professional are trained in the area of precision medicine, as the complexity of new drugs increases, the therapy choices available increase and our ability to delve deeper into making the right therapeutic choices for patients improves.

\section{FUNCTIONAL NEUROLOGICAL SYMPTOMS}

Tim Nicholson. Institute of Psychiatry, Psychology and Neuroscience, King's Medical School, London, UK

\subsection{6/postgradmedj-2018-fpm.10}

Functional Neurological Disorder (FND) is at the complex interface of the body and mind and therefore also physical and mental health. It has been neglected by medicine so little is known about its mechanism or treatment. However, there have been recent critical developments, particularly establishing it is as common as Parkinson's or multiple sclerosis and associated with poor outcomes and consequently associated with very high rates of disability and both health and social care costs.

There have also been critical changes in its diagnosis. The historical requirement of identifying causative stressors has been removed as it is now clear that these are not found in a significant proportion of patients. Instead, there has been increased emphasis on identifying 'positive neurological signs' which allow its reliable distinction from other disorders. Such signs can also be explained to patients to help them to understand the basis of the diagnosis and how it can be caused by dysfunction of cognitive processes such as attention and illness representations, thereby increasing acceptance of a complex and stigmatised diagnosis. The widespread adoption of the acronym FND and therefore the term 'functional', an aetiologically neutral term, has also facilitated critical steps in uniting patients and clinicians.

FND patients regularly have current and/or past comorbid symptoms presenting to other specialities such as fibromyalgia and Irritable Bowel Syndrome, which can be viewed as related disorders with overlapping mechanisms. Insights from FND research are therefore well positioned to inform the broader field of functional disorders which account for approximately $30 \%$ of morbidity and costs across medicine.

\section{REDUCING THE SEVERITY OF STROKE}

${ }^{1,2}$ Anthony Rudd. 'Stroke Consultant at St Thomas' Hospital, London and Professor of Stroke Medicine, King's College London, UK; ${ }^{2}$ National Clinical Director for Stroke with NHS England; London Stroke Clinical Director; Chair, Intercollegiate Stroke Working Party

\subsection{6/postgradmedj-2018-fpm.11}

100000 people in the UK have a stroke and worldwide it is responsible for 15 million deaths every year. With the ageing population, it is predicted that mortality will rise by $45 \%$ over the next 20 years in Europe and there will be over 4.5 million stroke survivors. Only 30 years ago it was considered to be a largely untreatable disease. There was no evidence for any acute intervention and rehabilitation was sporadic and rarely available at all in the community. In fact, little had changed over the previous century since Osler had written that 'there was no possibility of a cure'. Huge advances have been made in reducing the severity of stroke. Being managed on a stroke unit compared to a general ward reduces the odds ratio of death or dependency by $21 \%$. Intravenous thrombolysis does not save lives but overall greatly increases the chance of disability free survival. The last 3 years has seen publication of multiple trials of thrombectomy, all showing major benefits in reducing disability. Transferring patients from hospital to home early, continuing specialist rehabilitation and early supported discharge team are also more effective than conventional care at reducing the severity of stroke.

Delivering evidence based care to all patients regardless of where they live and when they have a stroke is challenging. Progress is being made with stroke being a priority area for the NHS, learning the lessons from the restructuring of stroke services in London and Manchester. 